\title{
On linear-quadratic optimal control problems for time-varying descriptor systems
}

\author{
Galina A.Kurina \\ Voronezh State Forestry Academy, \\ ul. Timirjazeva, 8 , \\ Voronezh, 394613, Russia \\ e-mail: kurina@kma.vsu.ru \\ phone: 7-0732-737148, fax: 7-0732-528610 \\ Roswitha März \\ Humboldt University \\ Institute of Mathematics \\ Sitz: Rudower Chaussee 25 \\ D-10099 Berlin, Germany \\ e-mail: iam@mathematik.hu-berlin.de \\ phone: +49-30-20932353 fax: +49-30-20932232
}

\begin{abstract}
The solvability of a linear-quadratic optimal control problem in a Hilbert space for time-varying descriptor systems is established under some conditions. Unlike previous works of other authors that are devoted to time-invariant descriptor systems in the finite-dimensional case we do not use the regularity of the pencil of the operators from the state equation, the factorability of the operator from the cost functional, and also the reduction of the operator preceding the derivative to a diagonal form.
\end{abstract}

\section{Introduction}

During the last twenty years many papers devoted to the study of optimal control problems for systems whose state equations are not resolved with respect to the derivative have been published (see, for example, reviews $[1,2]$ ). In the literature such systems are frequently called descriptor, implicit, singular, differential-algebraic, etc.

In the present paper the linear-quadratic optimal control problems for time-varying descriptor systems are considered under the most general conditions. Unlike previous works of other authors devoted to time-invariant descriptor systems in the finitedimensional case we do not use the regularity of the pencil of the operators from the state equation, the factorability of the operator from the cost functional, and also the reduction of the operator preceding the derivative to a diagonal form. 
The proof of the solvability is based on the form of the inverse operator to an operator $F: X_{1} \times X_{2} \times X_{3} \rightarrow X_{2} \times X_{1} \times X_{3}$, having a matrix representation of the following form

$$
F=\left(\begin{array}{rrr}
F_{1} & 0 & F_{2} \\
F_{3} & -F_{1}^{*} & F_{4} \\
-F_{4}^{*} & F_{2}^{*} & -F_{5}
\end{array}\right),
$$

where $X_{i}, i=\overline{1,3}$, are real Hilbert spaces, $F_{i}, i=\overline{1,5}$, are linear bounded operators acting in the appropriate spaces, $F_{3}, F_{5}$ are symmetric operators, i. e., $F_{3}=F_{3}^{*}, F_{5}=$ $F_{5}^{*}$, the exponent star with the notation of an operator denotes the adjoint operator. The operator of the form (1) is obtained by decomposing the system of equations from the optimality condition.

\section{Sufficient conditions of control optimality}

Let us consider the problem of minimizing the quadratic cost functional

$$
\begin{aligned}
J(u)= & \frac{1}{2}<x(T), V x(T)>+\frac{1}{2} \int_{0}^{T}(<x(t), W(t) x(t)> \\
& +2<x(t), S(t) u(t)>+<u(t), R(t) u(t)>) d t
\end{aligned}
$$

on trajectories of a linear system unresolved with respect to the derivative

$$
\begin{gathered}
\frac{d}{d t}(A(t) x(t))=C(t) x(t)+B(t) u(t), \\
A(0) x(0)=y_{0} .
\end{gathered}
$$

Here $t \in[0, T], T>0$ is fixed, $x(t) \in X, u(t) \in U ; y_{0} \in Y ; X, Y, U$ are real Hilbert spaces, $\langle\cdot, \cdot\rangle$ means a scalar product in appropriate spaces, $V, W(t) \in L(X), S(t) \in$ $L(U, X), R(t) \in L(U), A(t), C(t) \in L(X, Y), B(t) \in L(U, Y), W(t)=W^{*}(t), R(t)=$ $R^{*}(t)$ for all $t \in[0, T], V=V^{*}$, the operators $W(t), S(t), R(t), A(t), C(t), B(t)$ are continuous with respect to $t$.

Admissible controls are continuous functions with values in $U$ for which there is a solution of the problem (3), (4) (a solution of (3) is a continuous function $x:[0, T] \rightarrow X$ that has a continuously differentiable product $A(t) x(t)$ and satisfies (3) pointwise).

Assume the operator $A(t)$ to be normally solvable for all $t \in[0, T]$. Then the spaces $X$ and $Y$ are decomposed into the orthogonal sums $X=\operatorname{Ker} A(t) \oplus \operatorname{Im} A^{*}(t), Y=$ $\operatorname{Ker} A^{*}(t) \oplus \operatorname{Im} A(t)$. Denote by $P(t)$ the orthogonal projector of the space $X$ onto $\operatorname{Ker} A(t)$ and by $Q(t)$ the orthogonal projector of the space $Y$ onto $\operatorname{Ker} A^{*}(t)$. By $I$, as usually, we mean identity operators.

Remark 1: From the relation (4) it follows that $y_{0} \in \operatorname{Im} A(0)$, that is, for some $\tilde{x}_{0} \in X$ the equality $y_{0}=A(0) \tilde{x}_{0}$ should hold. Further we will assume that $y_{0} \in \operatorname{Im} A(0)$. Note that differential-algebraic systems (3) and initial value problems (3), (4) are considered e. g. in [7]. 
Let us assume that the following conditions are fulfilled.

I. The operator $V$ is positive semidefinite and $\operatorname{Im} V \subseteq \operatorname{Im} A^{*}(T)$.

II. For all $t \in[0, T]$ the operator $\left(\begin{array}{cc}W(t) & S(t) \\ S^{*}(t) & R(t)\end{array}\right)$ is positive semidefinite.

III. The projector $P(t)$ is continuous in $t$ and the projector $Q(t)$ depends smoothly on $t$.

Here we say that the operator $U \in L(X)$ is positive definite (semidefinite) if we have $<U x, x>>0(\geq 0)$ for all $x \in X$.

Lemma 1: If the conditions I., II. and III. are satisfied and the control $u_{*}(t)$ is a solution of the system

$$
\begin{gathered}
(A x)^{\prime}(t)=C(t) x(t)+B(t) u(t), \quad A(0) x(0)=y_{0}, \\
A^{*}(t)((I-Q) \psi)^{\prime}(t)=\begin{array}{l}
W(t) x(t)-\left(C^{*}(t)+A^{*}(t) Q^{\prime}(t)\right) \psi(t) \\
+
\end{array} \\
\quad S(t) u(t), A^{*}(T) \psi(T)=-V x(T), \\
0=-S^{*}(t) x(t)+B^{*}(t) \psi(t)-R(t) u(t),
\end{gathered}
$$

then $u_{*}(t)$ is an optimal control for the problem (2)-(4).

Proof: Let $x_{*}(t), u_{*}(t), \psi(t)$ be a solution of the system (5)-(7) (we assume that $\psi:[0, T] \rightarrow Y$ is a continuous function and $((I-Q) \psi)(t)$ is continuously differentiable), $u(t)$ is an arbitrary admissible control and $x(t)$ is a corresponding trajectory (a solution of the problem (3), (4)).

Taking into account the relations (2)-(7) after simple transformations we obtain the equality

$$
\begin{aligned}
J(u)-J\left(u_{*}\right)= & \frac{1}{2}<x(T)-x_{*}(T), V\left(x(T)-x_{*}(T)\right)> \\
& +\frac{1}{2} \int_{0}^{T}<\left(\begin{array}{c}
x(t)-x_{*}(t) \\
u(t)-u_{*}(t)
\end{array}\right),\left(\begin{array}{cc}
W(t) & S(t) \\
S^{*}(t) & R(t)
\end{array}\right)\left(\begin{array}{l}
x(t)-x_{*}(t) \\
u(t)-u_{*}(t)
\end{array}\right)>d t .
\end{aligned}
$$

By virtue of the conditions I., II., III. from the expression for $J(u)-J\left(u_{*}\right)$ it follows that $u_{*}(t)$ is an optimal control for the problem (2)-(4).

\section{Equations in subspaces}

Due to the Banach Theorem the operators

$$
\begin{aligned}
& A(t)=(I-Q(t)) A(t)(I-P(t)) \quad: \quad \operatorname{Im} A^{*}(t) \rightarrow \operatorname{Im} A(t), \\
& A^{*}(t)=(I-P(t)) A^{*}(t)(I-Q(t)) \quad: \quad \operatorname{Im} A(t) \quad \rightarrow \quad \operatorname{Im} A^{*}(t)
\end{aligned}
$$

have the bounded inverse operators $A^{+}(t)=(I-P(t)) A^{+}(t)(I-Q(t))$ and $A^{*+}(t)=(I-Q(t)) A^{+*}(t)(I-P(t))=A^{+*}$. 
Using the projectors $P, Q$ and the identities $Q(t)(A x)^{\prime}(t)=-Q(t) Q^{\prime}(t)(A x)(t)$, $Q(t)((I-Q) \psi)^{\prime}(t)=-Q(t) Q^{\prime}(t)((I-Q) \psi)(t),((I-P) x)(t)=\left(A^{+} A x\right)(t), Q^{\prime}(t)(I-$ $Q(t))=Q(t) Q^{\prime}(t)$ we obtain, from the system (5)-(7), the following system

$$
\begin{gathered}
0=L\left(\begin{array}{c}
A x \\
(I-Q) \psi
\end{array}\right)+G\left(\begin{array}{c}
P x \\
Q \psi \\
u
\end{array}\right), \\
\left(\begin{array}{c}
A x \\
(I-Q) \psi
\end{array}\right)^{\prime}=M\left(\begin{array}{c}
A x \\
(I-Q) \psi
\end{array}\right)+K\left(\begin{array}{c}
P x \\
Q \psi \\
u
\end{array}\right),
\end{gathered}
$$

where

$$
\begin{aligned}
L & =\left(\begin{array}{cc}
Q Q^{\prime}(I-Q)+Q C A^{+} & 0 \\
P W A^{+} & -P C^{*}(I-Q) \\
-S^{*} A^{+} & B^{*}(I-Q)
\end{array}\right), \\
G & =\left(\begin{array}{ccc}
Q C P & 0 & Q B \\
P W P & -P C^{*} Q & P S \\
-S^{*} P & B^{*} Q & -R
\end{array}\right), \\
\hat{M} & =\left(\begin{array}{cc}
(I-Q) C A^{+} & 0 \\
A^{*+} W A^{+} & -A^{*+} C^{*}(I-Q)
\end{array}\right), \\
M & =\hat{M}-\left(\begin{array}{cc}
Q Q^{\prime}(I-Q) & 0 \\
0 & Q Q^{\prime}(I-Q)
\end{array}\right),
\end{aligned}
$$

For brevity we will omit the argument $t$ in the notations.

With

$$
J=\left(\begin{array}{rr}
0 & I \\
-I & 0
\end{array}\right)
$$

and

$$
\hat{J}=\operatorname{diag}(J, I)
$$

it holds that

$$
K=J L^{*} \hat{J} .
$$

Following the lines of [7] one may easily check that (5)-(7) represents an index-1tractable DAE if and only if the operator $G(t)$ acts bijectively from Ker $A(t) \times$ Ker $A^{*}(t) \times U$ onto Ker $A^{*}(t) \times \operatorname{Ker} A(t) \times U=\operatorname{Im} G(t), \operatorname{Ker} G(t)=\operatorname{Im} A^{*}(t) \times \operatorname{Im} A(t) \times 0$.

Index-1 DAEs have nice solvability properties (cf. [7]). Consequently, the respective properties of the operator $G(t)$ are of great interest.

It is evident that the operator $G$ has a matrix representation of the form (1). Operators of the form (1) have interesting properties. 


\section{Properties of the operator (1)}

At first we establish some sufficient invertibility conditions for $F$.

Lemma 2: If the operator

$$
\left(\begin{array}{ll}
F_{3} & F_{4} \\
F_{4}^{*} & F_{5}
\end{array}\right)
$$

is positive definite and has the bounded inverse operator, if the operator $\left(F_{1} F_{2}\right)^{*}$ is injective and normally solvable, then $F$ has the bounded inverse operator.

Proof: Let us prove that there is no sequence $\left\{x_{n}\right\}$ such that $x_{n} \in X_{1} \times X_{2} \times X_{3},\left\|x_{n}\right\|=1$ for all $n \in N$ and $F x_{n} \rightarrow 0$.

We assume the contrary, namely

$$
\begin{aligned}
& F_{1} x_{n}^{1}+F_{2} x_{n}^{3} \rightarrow 0, \\
& F_{3} x_{n}^{1}-F_{1}^{*} x_{n}^{2}+F_{4} x_{n}^{3} \rightarrow 0, \\
&-F_{4}^{*} x_{n}^{1}+F_{2}^{*} x_{n}^{2}-F_{5} x_{n}^{3} \rightarrow 0,
\end{aligned}
$$

where $x_{n}=\left(\begin{array}{c}x_{n}^{1} \\ x_{n}^{2} \\ x_{n}^{3}\end{array}\right), x_{n}^{i} \in X_{i}, i=\overline{1,3},\left\|x_{n}\right\|=1$ for all $n \in N$. We scalarly multiply the left-hand side of the first relation of this system by $x_{n}^{2}$, the second relation by $x_{n}^{1}$ and the third relation by $\left(-x_{n}^{3}\right)$. Adding the results we obtain the relation

$$
\left\langle\left(\begin{array}{c}
x_{n}^{1} \\
x_{n}^{3}
\end{array}\right),\left(\begin{array}{ll}
F_{3} & F_{4} \\
F_{4}^{*} & F_{5}
\end{array}\right)\left(\begin{array}{c}
x_{n}^{1} \\
x_{n}^{3}
\end{array}\right)\right\rangle \rightarrow 0
$$

By virtue of the assumptions we have $x_{n}^{1} \rightarrow 0, x_{n}^{3} \rightarrow 0$.

Then the relation $\left(F_{1} F_{2}\right)^{*} x_{n}^{2} \rightarrow 0$ follows from the last system. Hence, we obtain $x_{n}^{2} \rightarrow 0$. This contradicts the equality $\left\|x_{n}\right\|=1$.

Therefore, there is a constant $k>0$ such that the inequality $\|F x\| \geq k$ is valid for all $x \in X_{1} \times X_{2} \times X_{3}$ with $\|x\|=1$. Further, we will use the inequality

$$
\|F x\| \geq k\|x\|,
$$

which is valid for all $x \in X_{1} \times X_{2} \times X_{3}$.

Now we prove that Ker $F^{*}=0$. From (1) it follows that the operator $F^{*}$ has the matrix representation

$$
F^{*}=\left(\begin{array}{rrr}
F_{1}^{*} & F_{3} & -F_{4} \\
0 & -F_{1} & F_{2} \\
F_{2}^{*} & F_{4}^{*} & -F_{5}
\end{array}\right) .
$$


Let us assume the contrary, i. e., there is a non-trivial element $x \in X_{2} \times X_{1} \times X_{3}$ such that $F^{*} x=0$. This equality is equivalent to the system

$$
\begin{aligned}
F_{1}^{*} x_{2}+F_{3} x_{1}-F_{4} x_{3} & =0, \\
-F_{1} x_{1}+F_{2} x_{3} & =0, \\
F_{2}^{*} x_{2}+F_{4}^{*} x_{1}-F_{5} x_{3} & =0,
\end{aligned}
$$

where $x=\left(\begin{array}{l}x_{2} \\ x_{1} \\ x_{3}\end{array}\right), x_{i} \in X_{i}, i=\overline{1,3}$.

We scalarly multiply the first equation of this system by $x_{1}$, the second equation by $x_{2}$ and the third equation by $\left(-x_{3}\right)$. Adding the obtained results yields the equality

$$
\left\langle\left(\begin{array}{r}
-x_{1} \\
x_{3}
\end{array}\right),\left(\begin{array}{ll}
F_{3} & F_{4} \\
F_{4}^{*} & F_{5}
\end{array}\right)\left(\begin{array}{r}
-x_{1} \\
x_{3}
\end{array}\right)\right\rangle=0
$$

By virtue of the assumption we have $x_{1}=0, x_{3}=0$.

Then the equality $\left(F_{1} F_{2}\right)^{*} x_{2}=0$ follows from the last system. We obtain $x_{2}=0$, hence a contradiction, therefore Ker $F^{*}=0$.

Let us prove that the range of values of the operator $F$ is a closed set. We take a sequence $\left\{y_{n}\right\}$, where $y_{n}=F x_{n}, x_{n} \in X_{1} \times X_{2} \times X_{3}, y_{n} \rightarrow y_{0}$. The sequence $\left\{F x_{n}\right\}$ is fundamental. From (12) it follows that the sequence $\left\{x_{n}\right\}$ is also fundamental. It tends to the element $x_{0} \in X_{1} \times X_{2} \times X_{3}$ by virtue of the completeness of $X_{i}$. As $F$ is a bounded operator, $F x_{n} \rightarrow F x_{0}$. Hence, $y_{0} \in \operatorname{Im} F$, i. e., $\operatorname{Im} F$ is the closed set.

Therefore, the following decomposition is realized: $X_{2} \times X_{1} \times X_{3}=\operatorname{Im} F \oplus \operatorname{Ker} F^{*}$. Since Ker $F^{*}=0$, using the inequality (12) we obtain that the operator $F$ has the bounded inverse operator (see, for example, Theorem 2 in [3], p. 204).

Lemma 2 is proved.

Remark 2: In the finite-dimensional case the conditions of Lemma 2 for the operator $G$ coincide with Assumption 2 in [4]. This assumption constitutes that part of the suppositions under which the linear-quadratic control problem with constant coefficients for descriptor systems was considered in [4] when $X=Y$.

Lemma 3: If the operator (11) is positive semidefinite and the operators

$$
\left(\begin{array}{lll}
F_{1} & F_{2}
\end{array}\right)^{*} \quad \text { and }\left(\begin{array}{cc}
F_{1} & F_{2} \\
F_{3} & F_{4} \\
F_{4}^{*} & F_{5}
\end{array}\right)
$$

are injective and normally solvable, then $F$ has the bounded inverse operator.

The proof of Lemma 3 is similar to the proof of Lemma 2.

Remark 3: It is easy to give an example showing that the conditions of the Lemma 2 and 3 are not necessary for the invertibility of any operator $F$ of the form (1), for 
example

$$
F=\left(\begin{array}{rrr}
1 & 0 & 0 \\
-1 & -1 & 0 \\
0 & 0 & 1
\end{array}\right)
$$

For the operator $F=\left(\begin{array}{lll}0 & 0 & 1 \\ 1 & 0 & 0 \\ 0 & 1 & 0\end{array}\right)$ (corresponding to the example in [4], p. 677) the conditions of Lemma 2 (Assumption 2 from [4]) are not satisfied, but the conditions of Lemma 3 are satisfied.

Lemma 4: If the operator $F$ is invertible, then the operator $\hat{J} F^{-1}$, where $\hat{J}$ : $X_{1} \times X_{2} \times X_{3} \rightarrow X_{2} \times X_{1} \times X_{3}$ has the matrix representation defined for $\hat{J}$ earlier, is symmetric.

Proof: We use the easily verifiable identity $F=-\hat{J} \tilde{F}$, where

$$
\tilde{F}=\left(\begin{array}{rrr}
F_{3} & -F_{1}^{*} & F_{4} \\
-F_{1} & 0 & -F_{2} \\
F_{4}^{*} & -F_{2}^{*} & F_{5}
\end{array}\right)
$$

is a symmetric operator. Thus, $\hat{J} F^{-1}=-\hat{J} \tilde{F}^{-1} \hat{J}^{-1}$ is the symmetric operator, because the operator $\tilde{F}^{-1}$ is symmetric and the operator $\hat{J}$ is unitary.

Corollary 1: If the operator $F$ is invertible, then the inverse operator $F^{-1}$ has a matrix representation of the form

$$
F^{-1}=\left(\begin{array}{rrr}
D_{1} & D_{2} & D_{3} \\
D_{4} & -D_{1}^{*} & D_{5} \\
D_{5}^{*} & -D_{3}^{*} & D_{6}
\end{array}\right)
$$

where $D_{2}, D_{4}, D_{6}$ are symmetric operators.

The Proof of this corollary follows from Lemma 4 and the matrix representation of the operator $\hat{J}^{-1}$.

Lemma 5: If the operator $F$ is invertible and the operator (11) is positive semidefinite, then the operators $\left(\begin{array}{rr}D_{2} & D_{3} \\ D_{3}^{*} & -D_{6}\end{array}\right)$ and $D_{4}$ from the representation (13) are positive semidefinite.

Proof: The proof of this Lemma is analogous of the proof of the similar Lemma for $F_{4}=0$ in [5]. Namely let us introduce the operator $\tilde{J}$ having the matrix representation

$$
\left(\begin{array}{ccc}
0 & I & 0 \\
I & 0 & 0 \\
0 & 0 & -I
\end{array}\right)
$$


For every $z=\left(\begin{array}{c}z_{2} \\ z_{1} \\ z_{3}\end{array}\right) \in X_{2} \oplus X_{1} \oplus X_{3}$ it is not difficult to verify the validity of the identity

$$
<\tilde{J} F^{-1} z, z>=<D_{4} z_{2}, z_{2}>+<\left(\begin{array}{cc}
D_{2} & D_{3} \\
D_{3}^{*} & -D_{6}
\end{array}\right)\left(\begin{array}{c}
z_{1} \\
z_{3}
\end{array}\right),\left(\begin{array}{c}
z_{1} \\
z_{3}
\end{array}\right)>
$$

Setting

$$
v=\left(\begin{array}{l}
v_{1} \\
v_{2} \\
v_{3}
\end{array}\right)=F^{-1} z
$$

we have

$$
<\tilde{J} F^{-1} z, z>=<\tilde{J} v, F v>=<\left(\begin{array}{cc}
F_{3} & F_{4} \\
F_{4}^{*} & F_{5}
\end{array}\right)\left(\begin{array}{l}
v_{1} \\
v_{3}
\end{array}\right),\left(\begin{array}{l}
v_{1} \\
v_{3}
\end{array}\right)>.
$$

Here $z, v$ are represented in the form corresponding to the matrix representation of the operator $F$.

Using the given condition we have the right-hand side of the identity (15), and hence the identity (14) is non-negative. Setting in (14) at first $z_{2}=0$ and secondly $z_{1}=$ $0, z_{3}=0$ we obtain the lemma statement.

\section{Solvability of the problem}

Now we turn back to the system (8), (9) resp. to the boundary value problem (5)-(7).

Theorem 1: Let the conditions I., II. and III. be satisfied. If, for every $t \in[0, T]$, the operator

$$
G(t): \operatorname{Ker} A(t) \times \operatorname{Ker} A^{*}(t) \times U \rightarrow \operatorname{Ker} A^{*}(t) \times \operatorname{Ker} A(t) \times U
$$

has the inverse operator $G(t)^{-1}$, then the system (8), (9) provides an explicit differential equation for the pair $(A x,(I-Q) \psi)$, that has the form

$$
\left(\begin{array}{c}
A x \\
(I-Q) \psi
\end{array}\right)^{\prime}=E\left(\begin{array}{c}
A x \\
(I-Q) \psi
\end{array}\right)-\left(\begin{array}{cc}
Q Q^{\prime}(I-Q) & 0 \\
0 & Q Q^{\prime}(I-Q)
\end{array}\right)\left(\begin{array}{c}
A x \\
(I-Q) \psi
\end{array}\right),
$$

where

$$
E=\left(\begin{array}{rr}
E_{1} & E_{2} \\
E_{3} & -E_{1}^{*}
\end{array}\right), \quad E_{2}=E_{2}^{*}, E_{3}=E_{3}^{*},
$$

the operators $E_{2}$ and $E_{3}$ are positive semidefinite.

Proof: Since $G(t)$ is invertible, from (8), (9) it follows immediately in view of the relation for $K$, that (16) holds true with $E=\hat{M}-J L^{*} \hat{J} G^{-1} L$. The term $\hat{J} G^{-1}$ is symmetric (cf. Lemma 4), thus $L^{*} \hat{J} G^{-1} L$ is also symmetric. Due to the structure of $\hat{M}$, we arrive at (17). 
It remains to show that the operators $E_{2}$ and $E_{3}$ in (17) are positive semidefinite. Let us write the expressions for the operators $E_{2}, E_{3}$.

$$
\begin{aligned}
E_{2}= & \left((I-Q) C P D_{2}-(I-Q) B D_{3}^{*}\right) P C^{*}(I-Q)-\left((I-Q) C P D_{3}\right. \\
& \left.+(I-Q) B D_{6}\right) B^{*}(I-Q), \\
E_{3}= & A^{*+} W A^{+}+\left((I-Q) Q^{\prime} Q+A^{*+} C^{*} Q\right)\left(D_{4}\left(Q Q^{\prime}(I-Q)+Q C A^{+}\right)\right. \\
& \left.-D_{1}^{*} P W A^{+}-D_{5} S^{*} A^{+}\right)+A^{*+} W P\left(-D_{1}\left(Q Q^{\prime}(I-Q)+Q C A^{+}\right)\right. \\
& \left.-D_{2} P W A^{+}+D_{3} S^{*} A^{+}\right)-A^{*+} S\left(D_{5}^{*}\left(Q Q^{\prime}(I-Q)+Q C A^{+}\right)\right. \\
& \left.-D_{3}^{*} P W A^{+}-D_{6} S^{*} A^{+}\right) .
\end{aligned}
$$

Here the operators $D_{i}, i=\overline{1,6}$, belong to the matrix representation (13) of $G^{-1}$ by Corollary 1.

For any $y \in Y$ we compute

$<E_{2}(I-Q) y,(I-Q) y>=<\left(\begin{array}{cc}D_{2} & D_{3} \\ D_{3}^{*} & -D_{6}\end{array}\right)\left(\begin{array}{c}P C^{*}(I-Q) y \\ -B^{*}(I-Q) y\end{array}\right),\left(\begin{array}{c}P C^{*}(I-Q) y \\ -B^{*}(I-Q) y\end{array}\right)>$.

Due to Lemma $5, E_{2}$ is positive semidefinite.

Next we turn to the operator $E_{3}$.

Taking into account the symmetry of the operators $D_{2}, D_{4}, D_{6}$ (see Corollarly 1 ) it is not difficult to verify the validity of the identities

$$
\begin{aligned}
& <E_{3}(I-Q) y,(I-Q) y>=<W A^{+}(I-Q) y, A^{+}(I-Q) y>-<D_{2} z_{1}, z_{1}> \\
& +<D_{4} z_{2}, z_{2}>+<D_{6} z_{3}, z_{3}>-2<D_{1}^{*} z_{1}+D_{5} z_{3}, z_{2}>+2<D_{3} z_{3}, z_{1}>= \\
& <\left(\begin{array}{cc}
W & S \\
S^{*} & R
\end{array}\right)\left(\begin{array}{c}
z_{4} \\
z_{5}
\end{array}\right),\left(\begin{array}{c}
z_{4} \\
z_{5}
\end{array}\right)> \\
& +<\left(D_{2}-D_{2} P W P D_{2}+D_{2} P S D_{3}^{*}+D_{3} S^{*} P D_{2}-D_{3} R D_{3}^{*}\right) z_{1}, z_{1}> \\
& +<\left(D_{4}-D_{1}^{*} P W P D_{1}-D_{1}^{*} P S D_{5}^{*}-D_{5} S^{*} P D_{1}-D_{5} R D_{5}^{*}\right) z_{2}, z_{2}> \\
& +<\left(-D_{6}-D_{3}^{*} P W P D_{3}-D_{3}^{*} P S D_{6}-D_{6} S^{*} P D_{3}-D_{6} R D_{6}\right) z_{3}, z_{3}> \\
& +2<\left(-D_{1}^{*} P W P D_{2}+D_{1}^{*} P S D_{3}^{*}-D_{5} S^{*} P D_{2}+D_{5} R D_{3}^{*}\right) z_{1}, z_{2}> \\
& +2<\left(-D_{3}^{*}+D_{3}^{*} P W P D_{2}-D_{3}^{*} P S D_{3}^{*}+D_{6} S^{*} P D_{2}-D_{6} R D_{3}^{*}\right) z_{1}, z_{3}> \\
& +2<\left(D_{3}^{*} P W P D_{1}+D_{3}^{*} P S D_{5}^{*}+D_{6} S^{*} P D_{1}+D_{6} R D_{5}^{*}\right) z_{2}, z_{3}>,
\end{aligned}
$$

where $z_{1}=P W A^{+}(I-Q) y, z_{3}=\left(Q Q^{\prime}(I-Q)+Q C A^{+}\right)(I-Q) y, z_{3}$ $=S^{*} A^{+}(I-Q) y, z_{4}=A^{+}(I-Q) y+P\left(-D_{2} z_{1}-D_{1} z_{2}+D_{3} z_{3}\right), z_{5}=D_{3}^{*} z_{1}-D_{5}^{*} z_{2}+D_{6} z_{3}$, the argument $t$, as well as earlier, is omitted everywhere for brevity of an entry.

Using Corollary 1 and the identities

$$
\begin{aligned}
& Q C P D_{1}+Q B D_{5}^{*}=I, Q C P D_{2}-Q B D_{3}^{*}=0, Q C P D_{3}+Q B D_{5}=0, \\
& P W P D_{1}-(Q C P)^{*} D_{4}+P S D_{5}^{*}=0, P W P D_{2}+(Q C P)^{*} D_{1}^{*}-P S D_{3}^{*}=I, \\
& P W P D_{3}-(Q C P)^{*} D_{5}+P S D_{6}=0, \\
& -S^{*} P D_{1}+B^{*} Q D_{4}-R D_{5}^{*}=0,-S^{*} P D_{2}-B^{*} Q D_{1}^{*}+R D_{3}^{*}=0, \\
& -S^{*} P D_{3}+B^{*} Q D_{5}-R D_{6}=I,
\end{aligned}
$$


following from the definition of the operator $G^{-1}$ we obtain that all scalar products in the expression for $<E_{3}(I-Q) y,(I-Q) y>$, excepting the first, are identically equal to zero (for example we have $D_{2}-D_{2} P W P D_{2}+D_{2} P S D_{3}^{*}+D_{3} S^{*} P D_{2}-D_{3} R D_{3}^{*}=$ $\left.D_{2}-D_{2}\left(I-(Q C P)^{*} D_{1}^{*}\right)+D_{3}\left(-B^{*} Q D_{1}^{*}\right)=\left(D_{2}(Q C P)^{*}-D_{3} B^{*} Q\right) D_{1}^{*}=0\right)$.

Theorem 1 is proved.

Remark 4: If the continuously differentiable functions $y:[0, T] \rightarrow Y, z:[0, T] \rightarrow Y$ satisfy the explicit differential equation (cf. (16))

$$
\left(\begin{array}{l}
y \\
z
\end{array}\right)^{\prime}=E\left(\begin{array}{l}
y \\
z
\end{array}\right)-\left(\begin{array}{cc}
Q Q^{\prime}(I-Q) & 0 \\
0 & Q Q^{\prime}(I-Q)
\end{array}\right)\left(\begin{array}{l}
y \\
z
\end{array}\right)
$$

then, multiplying (18) by $\operatorname{diag}(Q, Q)$ and taking into account that $\operatorname{diag}(Q, Q) E=$ $0, Q^{\prime}(I-Q)=Q Q^{\prime}$, we find that

$$
\left(\begin{array}{l}
Q y \\
Q z
\end{array}\right)^{\prime}=-\left(\begin{array}{ll}
(I-Q) Q^{\prime} Q & 0 \\
0 & (I-Q) Q^{\prime} Q
\end{array}\right)\left(\begin{array}{l}
Q y \\
Q z
\end{array}\right)
$$

holds true. Consequently, if $Q\left(t_{0}\right) y\left(t_{0}\right)=0, Q\left(t_{0}\right) z\left(t_{0}\right)=0$, for a certain $t_{0} \in[0, T]$, then $Q(t) y(t), Q(t) z(t)$ vanish identically, i. e. $\operatorname{Im} A(t) \times \operatorname{Im} A(t) \subseteq Y \times Y$ becomes a time-varying invariant subspace of the differential equation (18). Since (19) decouples into $(Q y)^{\prime}=(I-Q) Q^{\prime} Q(Q y),(Q z)^{\prime}=(I-Q) Q^{\prime} Q(Q z), y(0) \in \operatorname{ImA}(0), z(T) \in$ $\operatorname{Im} A(T)$ also imply $y(t) \in \operatorname{Im} A(t), z(t) \in \operatorname{Im} A(t), t \in[0, T]$, and vice versa.

The boundary conditions in (5), (6) lead to $y(0)=A(0) x(0)=y_{0} \in \operatorname{Im} A(0), z(T)=$ $(I-Q(T)) \psi(T) \in \operatorname{Im}\left(A^{*+}(T) V\right)$, due to $\operatorname{Im} V \subseteq \operatorname{Im} A^{*}(T)$. Therefore, only those solutions of (18) which belong to the mentioned invariant subspace are relevant for the solution of the system (5)-(7).

In case, this invariant subspace does not vary with $t$, i. e. if $\operatorname{Im} A(t)=\operatorname{Im} A(0)$ for all $t \in[0, T]$ and hence the projector $Q(t)$ is constant, things become easier. Then, (18) simplifies to

$$
\left(\begin{array}{l}
y \\
z
\end{array}\right)^{\prime}=E\left(\begin{array}{l}
y \\
z
\end{array}\right)
$$

on the constant space $\operatorname{Im} A(0) \times \operatorname{Im} A(0)$.

Theorem 2: Under the conditions of Theorem 1, if $\operatorname{Im} A(t)$ is invariant with respect to $t$, the system (8), (9) is reduced to the non-negative Hamiltonian system resolved with respect to the derivative.

Remark 5: For operators $A(t), R(t)$ that have bounded inverses $A^{-1}(t), R^{-1}(t)$ respectively on $Y, U$, and in case of $S(t)=0, t \in[0, T]$, the equation (16) has the special form

$$
\left(\begin{array}{c}
A x \\
\psi
\end{array}\right)^{\prime}=\left(\begin{array}{cc}
C A^{-1} & B R^{-1} B^{*} \\
A^{*-1} W A^{-1} & -A^{*-1} C^{*}
\end{array}\right) \quad\left(\begin{array}{c}
A x \\
\psi
\end{array}\right) .
$$


Obviously, this is a non-negative Hamiltonian system. If, additionally, $A(t)$ depends smoothly on $t$, we might turn to an explicit differential equations with respect to the unknowns $x, \psi$, but the resulting system

$$
\left(\begin{array}{c}
x \\
\psi
\end{array}\right)^{\prime}=\left(\begin{array}{cc}
A^{-1} C-A^{-1} A^{\prime} & A^{-1} B R^{-1} B^{*} \\
A^{*-1} W & -A^{*-1} C^{*}
\end{array}\right) \quad\left(\begin{array}{c}
x \\
\psi
\end{array}\right)
$$

is no more Hamiltonian in general. This is why we should prefer the form (20) also in this case. It should be mentioned that, if we consider the new variable $y=A^{*} \psi$ then from the last system we obtain the non-negative Hamiltonian system with respect to the unknowns $x, y$.

The Theorem 2 allows to apply results on the unique resolvability of two-point boundary values problem, which are known for non-negative Hamiltonian systems, to systems of the form (5)-(7).

For example, we have the theorem stated below.

Theorem 3: If, for every $t \in[0, T]$, the operator $G(t)$ has the inverse operator on the space $\operatorname{Ker} A^{*}(t) \times \operatorname{Ker} A(t) \times U$, the conditions I., II., III. are satisfied, $\operatorname{Im} A(t)$ does not depend on $t$, then the problem (2)-(4) is resolvable.

Remark 6: The Theorems 2 and 3 are also true if $\operatorname{ImA}(t)$ depends on $t$ but $Q Q^{\prime}(I-Q) \equiv 0$.

Now we investigate the solvability of the equation (18) with the boundary values

$$
y(0)=y_{0}, z(T)=-A^{*+}(T) V A^{+}(T) y(T)
$$

in the finite-dimensional case.

Theorem 4: If the space $Y$ is finite-dimensional then the equation (18) with the boundary values (21) has the unique solution.

Proof: It is enough to prove that the problem

$$
\begin{aligned}
& x_{1}^{\prime}=E_{1} x_{1}+E_{2} x_{2}-Q Q^{\prime}(I-Q) x_{1}, \\
& x_{2}^{\prime}=E_{3} x_{1}-E_{1}^{*} x_{2}-Q Q^{\prime}(I-Q) x_{2}, \\
& x_{1}(0)=0, \\
& x_{2}(T)=-\tilde{V} x_{1}(T),
\end{aligned}
$$

where $\tilde{V}=A^{*+}(T) V A^{+}(T)$, has only the zero solution.

We scalarly multiply the first equation of (22) by $x_{2}$ and the second equation by $x_{1}$. Adding the results and taking into account Remark 4 we obtain the relation $<x_{1}, x_{2}>^{\prime}=<E_{2} x_{2}, x_{2}>+<E_{3} x_{1}, x_{1}>$.

Integrating the last equation on the segment $[0, T]$ in view of the boundary values we have $\left\langle x_{1}(T), \tilde{V} x_{1}(T)>+\int_{0}^{T}\left(<E_{2} x_{2}, x_{2}>+<E_{3} x_{1}, x_{1}>\right) d t=0\right.$. 
Since the self-adjoint operators $\tilde{V}, E_{2}, E_{3}$ are positive semidefinite (see Theorem 1) and the operators $E_{2}, E_{3}$ are continuous with respect to $t$ then from the previous relation it follows that $E_{2} x_{2} \equiv 0, E_{3} x_{1} \equiv 0$.

From here and from the system (22) we have $x_{1} \equiv 0, x_{2} \equiv 0$.

Theorem 4 is proved.

From Lemma 1, Theorems 1, 4 we obtain the following theorem, which generalizes Theorem 3 (for finite-dimensional $Y$ ) to time-varying $\operatorname{Im} A(t)$.

Theorem 5: If the space $Y$ is finite-dimensional and if for every $t \in[0, T]$ the operator $G(t)$ has the inverse operator on the space $\operatorname{Ker} A^{*}(t)$ x $\operatorname{Ker} A(t)$ x $U$, the conditions I, II, III are satisfied then the problem (2)-(4) is resolvable.

Remark 7: Some results of the present paper for $A(t)$ being constant with respect to $t$ are given in [5] $(S=0)$ and in [6] $(S \neq 0)$.

Linear-quadratic problems with constant coefficients for continuous descriptor systems were considered in [4] in the finite-dimensional case if $X=Y$ by means of a different approach. Let us stress that here we do not use the regularity of the pencil $\mu A-C$, the reduction of $A$ to a diagonal form and the factorability of the operator from the cost. Moreover, the realization of Assumption 2 from [4] for the operator $G$ is not mandatory here.

\section{References}

[1] F. L. Lewis:A survey of linear singular systems. Circuits, Systems, and Signal Processing. 1986. V. 5. No. 1, p. 3-36.

[2] G. A. KuRINA: Singular perturbations of control problems with state equation unresolved with respect to derivative. Survey. Izvestija RAN. Tehnicheskaja kibernetika. 1992. No. 4, p. 20-48 (in Russian).

[3] L. V. Kantorovich, G. P. Akilov: Funkcional'nyj analiz. M. Nauka, 1984 (in Russian).

[4] D. J. Bender, A. J. Laub: The linear-quadratic optimal regulator for descriptor systems. IEEE Transactions on Automatic Control. 1987. V. 32. No. 8, p. $672-688$.

[5] G. A. Kurina: To theory of linear-quadratic control problems unresolved with respect to derivative. Funkcional'nye prostranstva i uravnenija matematicheskoi fiziki. Sbornik statei. Voronezh, izdatel'stvo VGU. 1988. p. 25-32. See also the unpublished paper VINITI No. 755-B86, 1986 (in Russian).

[6] G. A. KuRINA: On linear-quadratic control problems for descriptor systems. International Conference "Dynamical Systems: Stability, Control, Optimization" (DSSCO’98). Abstracts. Volume 2. Minsk, Belarus, 1998, p. 170-172 (in Russian).

[7] K. BAlla, R. MÄrZ: Linear differential algebraic equations of index 1 and their adjoint equations. Results in Mathematics 2000, V. 37, p. 13-35. 